\title{
Accidental barium bronchography
}

\author{
Ahmad Zaheen MD MSc, Matthew B. Stanbrook MD PhD
}

- Cite as: CMAJ 2017 May 8;189:E670. doi: 10.1503/cmaj.160969

A 46-year-old man with a history of $\mathrm{T} 2 \mathrm{~N} 2$ right tonsillar squamous cell carcinoma was referred to the emergency department after a large aspiration event during a barium esophogram. His cancer had been treated five years previously with panitumumab, radiation and salvage surgery for residual tumour. The esophogram was arranged for a six-month history of dysphagia and periprandial coughing up of food contents. The patient was asymptomatic upon arrival in the emergency department.

Upon examination, he appeared well and hemodynamically stable, with a respiratory rate of 20 breaths per minute, oxygen saturation of $94 \%$ on ambient air and a temperature of $38.1^{\circ} \mathrm{C}$. Respiratory examination showed no increased work of breathing, with crackles in the right lung base on auscultation. Neurologic deficits included palsy of the right hypoglossal nerve and asymmetry of the soft palate. Chest radiographs conducted in the emergency department showed accumulation of barium in the right middle and lower lobes (Figure 1). Bronchoscopy confirmed localization of barium in the right lung, with no evidence of tracheoesophageal fistula. Nasopharyngoscopy showed normal vocal cord function, but absent supraglottic and glottic sensation. A videofluoroscopic swallowing examination identified uncoordinated swallowing with florid aspiration, and a diagnosis of aspiration pneumonitis was made. These findings were attributed to delayed effects from radiation treatment. He remained clinically stable and a feeding gastrostomy tube was inserted.

Risk factors for aspiration include altered level of consciousness, neurologic dysfunction of the upper airway, structural esophageal disease, cancers of the aerodigestive tract and iatrogenic disruption of the airway (i.e., intubation and endoscopy). ${ }^{1}$ The anatomic configuration of the bronchial tree predisposes contents to enter the right and middle lobes on erect aspiration, as shown in our case.

Aspiration pneumonitis is an inflammatory injury to the lung triggered by exposure to noxious stimuli, typically of gastric origin. Clinical manifestations range from none to cough, wheeze, dyspnea, respiratory failure and, in severe cases, acute respiratory dis- tress syndrome. ${ }^{1}$ Treatment is typically supportive, and routine antibiotic use is not indicated. ${ }^{1}$

Barium is chemically inert and had been used as a contrast medium for bronchography before the advent of computed tomography and bronchoscopy. ${ }^{2}$ Although historically found to be safe in this context, aspiration of barium may also produce a chemical pneumonitis in some cases. ${ }^{3}$

\section{References}

1. Marik PE. Aspiration pneumonitis and aspiration pneumonia. N Engl J Med 2001;344:665-71.

2. Shook CD, Felson B. Inhalation bronchography. Chest 1970;58:333-7.

3. Hundemer GL, Kumar V, Vaduganathan M. Large-volume barium aspiration. Proc Bayl Univ Med Cent 2015;28:183-4.

Competing interests: None declared.

This article has been peer reviewed.

The authors have obtained patient consent.

Affiliations: Department of Medicine (Zaheen), University of Toronto; Division of Respirology (Stanbrook), Toronto Western Hospital, Toronto, Ont.

Disclaimer: Matthew Stanbrook is a deputy editor for CMAJ and was not involved in the editorial decision-making process for this article.

Correspondence to: Matthew Stanbrook, matthew.stanbrook@uhn.ca 